\title{
Ricerche di ottica e di spettroscopia nell' alta atmosfera $\left(^{*}\right)$
}

\author{
R. Cialdea
}

Ricevuto il 6 Novembre 1964

Riassunto. - L'A. fa un panorama delle ricerche di ottica e di spettroscopia dell'alta atmosfera, sottolineandone tre aspetti particolari:

1) studio delle proprietí fisiche dell'atmosfera mediante l'osservazione spettroscopica delle bande di assorbimento della radiazione solare e della radiazione del cielo notturno.

2) studio degli oggetti celesti considerati come sorgenti di radiazione ultravioletta e di raggi $\mathrm{X}$.

3) diminuzione della visibilità diurna di oggetti extraterrestri (stelle, corona solare...) a causa della diffusione della luce da parte dell'atmosfera. lazione.

Questo panorana è linitato al 1962, anno in cui è stata tenuta la re-

SUMMARY. - The A. makes a review of the optical and spectroscopical researches in the higher atmosphere, in regard to the following aspects:

1) the investigation of the physical properties of the atmosphere by the spectroscopical examination of the absorption bands and the night sky radiations.

2) the investigation of the celestial bodies as the ultraviolet and $\mathrm{X}$-rays sources.

3) the investigation of the visibility decrease of the extraterrestrial objects (the stars, the solar corona...) because of the light scattering of the atmosphere.

This roview arrives until the meeting's date (1962).

Jo scopo della presente relazione è l'illustrazione di alcuni aspetti delle ricerche di ottica e di spettroscopia nell'alta atmosfera. Si tratta di un campo quanto mai vasto e qui non potrò dare altro che un rapido panorama sui problemi più importanti.

$\left(^{*}\right)$ Relazione Generale al $2^{\circ}$ Congresso Intemazionale Tecnico-Scientifico dello Spazio, Roma, 19.23 Giugno 1962. 
I principali fini che si prefiggono i fisici, $i$ geofisici e gli astrofisici in questa ricerche sono tre e cioè:

A. - Proprietà fisiche dell'atmosfera con la determinazione della. distribuzione verticule e delle densità dei diversi componenti dellaria e della temperatura. Ciò viene eseguito osservando spettroscopicamente di giorno la radiazione solare parzialmente assorbita dall'atmosfera terrestre, e di notte la radiazione emessa dagli strati dell'atmosfera stessa.

B. - Studio della radiazione emessa dagli oggetti celesti e in particolar modo dal sole, nel campo dell'u.v. e dei raggi $\mathbf{X}$.

C. - Visibilità di oggetti extraterrestri, con lo studio delle due cause che la alterano:

a) la rifrazione attraverso l'atmosfera con gli effetti di scintillazione e di deviazione.

b) la diffusione della luce con la conseguente invisibilità diurna di oggetti molto deboli, quali le stelle, la corona solare ecc.

In questi ultimi anni l'interesse per lo studio degli alti strati dell'atmosfera è andato via via aumentando, data l'attuale meta che l'uomo si è prefissa, la realizzazione cioè dei viaggi interplanetari. Ma l'interesse puramente scientifico della conoscenza dell'alta atmosfera ha spinto da più di un secolo i fisici, i geofisici e gli astronomi a scandagliarla con tutti i mezzi a loro disposizione: cosi abbiamo avuto un gracluale svilupparsi di metodi e di tecniche di osservazione.

E chiaro che il metorlo più sicuro per determinare le caratteristiche dell'atmosfera alle varie quote è quello diretto per mezzo di palloni, di razzi, di satelliti. Ma questi metodi hamno un loro intrinseco difetto; essi si limitano ad analizzare una piccola porzione dell'atmosfera in vicinanza della loro traiettoria e della loro orbita e, soprattutto, durante un intervallo di tempo molto breve. Anche per i satelliti, che possono rimanere in orbita per molto tempo, la loro velocità è tale e le loro orbite sono così diverse l'una dall'altra che l'analisi da loro eseguita non puó riferirsi praticamente che a brevi intervalli di tempo. E ben noto invece che ogni fenomeno atmosferico subisce delle variazioni, non sempre regolari, sia diurne che stagionali, senza parlare poi delle fluttuazioni inevitabili dovute alle infinite cause che possono generare delle perturbazioni.

Ma questi metodi sono peró insostituibili per il loro pregio di poter fornire dei dati locali sicuri sulle proprietà fisiche dell'atmosfera.

Gli altri metodi invece sono esclusivamente basati su misure effettuate a.l suolo. Questi metodi hanno lo svantaggio di non poter fornire 
che dei valori integrati a tutta l'atmosfera e quindi non possono permettere una sicuma determinazione delle proprietà locali alle diverse quote.

Ho parlato di «sicura determinazione » in quanto in alcuni casi e sotto alcune ipotesi restrittive è possibile calcolare, con reiterate misure la distribuzione verticale di queste proprietà. Ma è necessario tener presente che la distribuzione verticale cosi ottenuta è il risultato di calcoli basati su ipotesi non sempre universalmente accettabili.

Il vantaggio di un tale metodo è quello di poter moltiplieare gli osservatori e di poter eseguire le misure in modo continuo su vastissime regioni della terra, a parte ovviamente le difficoltà di indole organizzativa.

Devo infine far osservare che qualora si tratti di eseguire delle ricerche in regioni spettrali con $\lambda<2900 \AA$, è inevitabile l'uso di razzi o di satelliti in quanto l'atmosfera al suolo a partire da $2900 \AA$ diventa praticamente opaca.

Passeró ora in un rapida rassegna i tre sopramenzionati campi di ricerca, soffermandomi in particolar modo sui metodi adoperati.

A1. - Il primo vastissimo campo di ricerche riguarda anzitutto l'assorbimento della mdiazione solare da parte dei gas costituenti l'atmosfera. Ogni gas ha un proprio spettro di assorbimento e la radiazione as-

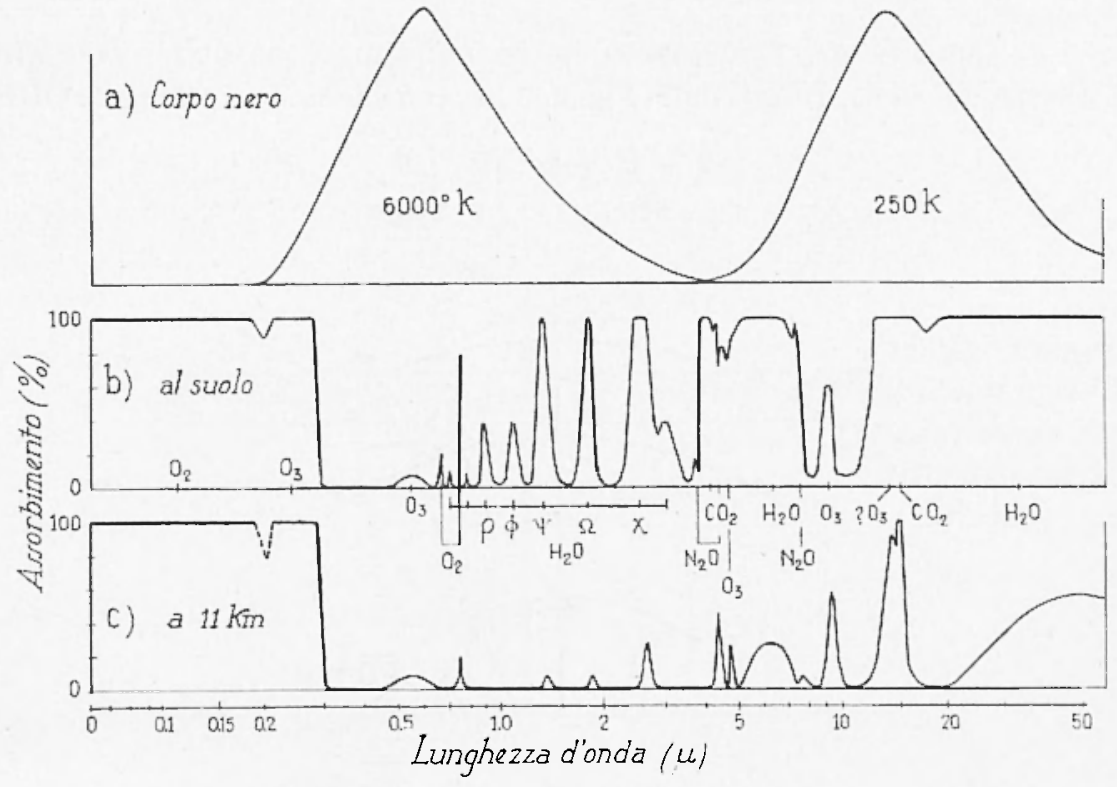

Fig. $I(*)$

$\left({ }^{*}\right)$ Da R. M. Goowy (1) p. 145. 
sorbita da esso in una certa riga o in una certa banda è tanto più grande, quanto maggiore è la massa del gas attraversata: da qui discende la possibilità di fare un'analisi quantitativa (spettrofotografica o spettrometrica) dei gas o dei rapori presenti nell'atmosfera. Questo metodo è stato applicato per la valutazione del contenuto nell'atmosfera di alcuni componenti e principalmente dell'ozono, del vapor d'ac'qua, dell'anidride ('arbonica.

La misura viene effettuata misumando l'intensità della radiazione che ci perviene dal sole, al centro della banda di assorbimento ed immediatamente al di fuori di essa.

Nella Fig. 1 è riportato lo spettro di assorbimento con le bande caratteristiche di alcuni componenti dell'atmosfera, con i valori dell'assorbimento osservato al livello del suolo e a $11 \mathrm{~km}$.

E possibile inoltre, applicando dei particolari metodi, avere un'idea della distribuzione verticale di un dato componente dell'atmosfera. Accennerò solo ai tre metodi seguenti:

a) l'ipotesi dello strato sottile;

b) l'Umkehr-eftekt;

c) le ecolissi di luna.

a) Con l'ipotesi dello strato sottile uniforme d̀ possibile conoscere l'altezza dal suolo. Infat ti dalla Fig. 2 , si ha, considerando il triangolo OBP

$$
\frac{R+H+h o}{\operatorname{sen} \zeta}=\frac{R}{\operatorname{sen} a}
$$

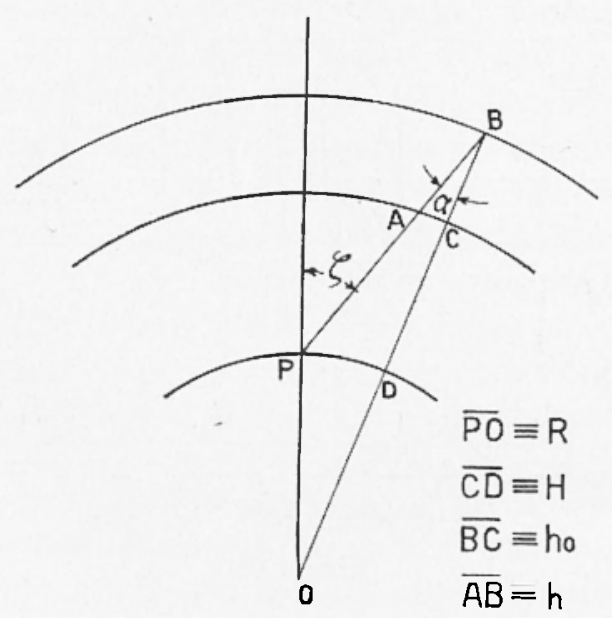

Fig. 2 
e dal triangolo mistilineo $\triangle \mathrm{BC}$, in prima approssimazione

e quindi

$$
h=\frac{h_{n}}{\cos \alpha}
$$

$$
h=\frac{h_{0}}{\sqrt{1-\left(\frac{R}{R+H+h_{0}}\right)^{2} \operatorname{sen}^{2} \zeta}}
$$

e con l'ipotesi

$$
\begin{gathered}
h_{0} \ll R+H \\
h=\frac{h_{0}}{\sqrt{1-\left(\frac{R}{R+H}\right)^{2} \operatorname{sen}^{2} \zeta}}
\end{gathered}
$$

dove $R$ è il raggio della terra, $H$ l'altezza dello strato, $\zeta$ l'altezza zenitale del sole.

Poiché l'assorbimento dipende dalla massa del gas assorbente attraversat o e quindi dallo spessore $h$, applicando più volte la [1] per distanze zenitali diverse è possibile ricavare $H$, cioc̀ l'altezza dello strato.

b) L'Umkher-effekt. E l'effetto che si osserva quando si misura la radiazione diffusa allo zenith (Fig. 3), per due lunghezze d'onda per le quali

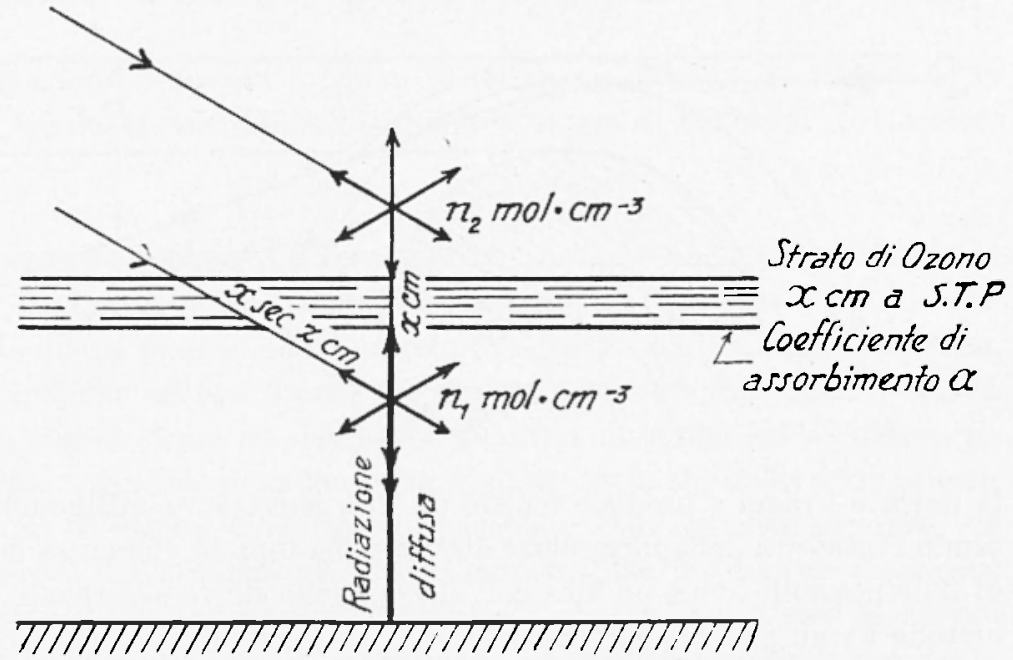

Fig. $3\left(^{*}\right)$

(*) Da R. H. Goody ( $\left.{ }^{1}\right)$ 1). 92. 
un componente dell'atmosfera, per es. l'ozono, presenta due coefficienti di assorbimento diversi. Siano $\lambda_{1}$ e $\lambda_{2}$ le lunghezze d'onda scelte per le quali i coefficienti di assorbimento sono $a_{1}$ et $a_{2}$ rispettivamente, con $a_{1}>a_{2}$. Man mano che il sole scende sull'orizzonte il rapporto $\frac{I_{\lambda_{1}}}{I_{\lambda_{2}}}$ diminuisce fino a raggiungere un minimo (per angoli zenitali del sole di circa $85^{\circ}$ ) per poi risalire. Questo fenomeno dell'inversione (Umkher) dell'andamento del rapporto $\frac{I_{\lambda_{1}}}{I_{\lambda_{2}}}$ dipende dal fatto che la radiazione diffusa proviene principalmente dalle due regioni dell'atmosfera che si trovano al di sopra ed al di sotto dello strato di ozono. Mentre la $\lambda_{2}$ (con $\alpha_{2}$ più piccolo) viene diffusa da ambedue le regioni, la $\lambda_{1}$ (con $\alpha_{1}$ più grande) viene, per grandi angoli zenitali, diffusa quasi esclusivamente dalla regione superiore. Infatti dato il forte valore dell'assorbimento, lo strato di ozono, a partire da un certo valore della distanza zenitale del sole, diventa praticamente opaco.

L'andamento dell'Umkher-effekt dà la possibilità di determinare la distribuzione verticale dell'ozono con una certa approssimazione.

c) Le eclissi di luna. Durante un'eclisse di luna i raggi del sole, sfiorando la terra possono arrivare sulla superficie lunare. Se non ci fosse l'atmosfera l'ombra della terra sarebbe data da $W$ (Fig. 4). Ma l'atmosfera

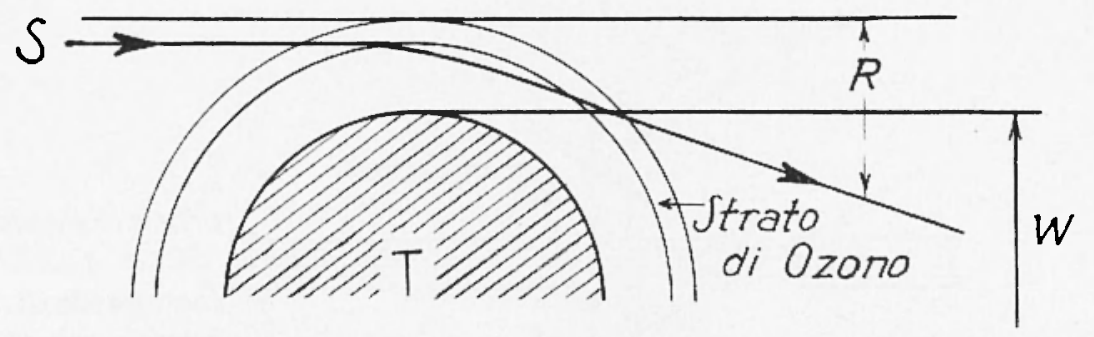

Fig. $4(*)$

fa deviare i raggi e produce inoltre un loro parziale assorbimento. Misurando l'intensità della luce solare diffusa dalla luna in vicinanza del bordo di $W$, ̀̀ possibile avere un'idea dell'altezza dello strato assorbente. Questo metodo ha un grave inconveniente che ne limita la pressione, dovuto al fatto che il sole ha un diametro di circa $32^{\prime}$.

(*) Da H.S. W. Massey e R. L. F. Boyd $\left(^{2}\right)$ p. 133. 
Infine desidero accennare alla importanza meteorologica della determinazione dell'altezza dello strato di ozono. Ia formazione dell'ozono è regolata da una doppia reazione (I + II).

I. - Formazione di ossigeno atomico per fotodissociazione secondo due reazioni

$$
O_{2}+h \nu \rightarrow O\left({ }^{3} P\right)+O\left({ }^{1} D\right)
$$

dovuta a fotoni di lunghezza d'onda $\lambda<1760 \AA$ : questo assorbimento genera una larga banda chiamata il continuo di Schumann-Runge. L'altra reazione è

$$
\mathrm{O}_{2}+h \nu \rightarrow 2 O\left({ }^{3} \mathrm{P}\right)
$$

dovuta a fotoni di lunghezza d'onda $\lambda<2420 \AA$ : questo assorbimento genera l'altra banda chiamata continuo di Herzberg.

II. - Formazione di ozono secondo la reazione

$$
\mathrm{O}+\mathrm{O}_{2}+\mathrm{M} \rightarrow \mathrm{O}_{3}+\mathrm{M}
$$

dove $M$ è un atomo o una molecola che ha la funzione di assorbire l'energia in eccesso.

D'alta parte si hanno altre due reazioni che tendono a distruggere l'ozono:

$$
O_{3}+h v \rightarrow O_{2}+O\left({ }^{1} D\right)
$$

questa reazione è dovuta a fotoni di Iunghezza d'onda $\lambda<2550 \AA$ : la relativa banda di assorbimento prende il nome di continuo di Hartley.

$$
O_{3}+h v \rightarrow O_{2}+O\left({ }^{3} P\right)
$$

questa reazione è dovuta a fotoni di lunghezza d'onda $\lambda<6100 \AA$ : la relativa banda prende il nome di continuo di Chappuis.

Date queste reazioni fotochimiche che genemo e distruggono l'ozono, ne consegue che ad ogni quota noi dovremmo avere una certa quantità di ozono che si forma ed una certa quantità di ozono che si distrugge. Queste due quantità dipendono alle diverse quote sia dalle relative concentrazioni di ozono, di ossigeno molecolare e di ossigeno atomico, sia dall'intensità della ladiazione che vi giunge; questa intensità dipenderà ovviamente da tutti i processi fotochimici che sono avvenuti al disopra della quota considerata. Ad ogni quota corrisponderà quindi una certa concentrazione di equilibrio di ozono. E presumibile che essa presenterà almeno un massimo, che dipenderà certamente dalla pressione. A titolo di esempio citerò due regole valevoli in generale alle nostre latitudini. 
1. - La quantità di ozono tende a diminuire all'avvicinarsi di un fronte callo: ciò può risentirsi ad una distanza che può raggiungere anche i mille chilometri.

2. - La quantità di ozono tende ad aumentare quando si ha un passaggio di un fronte freddo. Purtroppo le osservazioni sono ancora insufficienti per poter dare delle regole attendibili per le variazioni di altezza. Ma è cepto che deve esserei una correlazione tra la quota della massima concentrazione dell'ozono ed i movimenti verticali dell'atmosfera.

I problemi riguardanti gli altri componenti sono analoghi, a parte il fatto che essi risentono molto delle condizioni locali, a differenza dell'ozono che dipende sostanzialmente dalla concentrazione di ossigeno, che, a parte le variazioni dovute alle variazioni di pressione, sono rigorosamente uguali in ogni punto della terra.

A2. - Il secondo campo di ricerche riguarda invece lo spettro di emissione dell'atmosfera. E noto che il cielo di notte presenta una luminosità gencealmente molto bassa; essa è dovuta al fatto che una certa quantita di atomi o molecole vengono a trovarsi in uno stato eccitato o per urti o per assorbimento di fotoni.

Di solito si usa distinguere due tipi di emissione:

a) luce aurorale: essa è dovuta agli atomi (o alle molecole) eccitate dalla radiazione corpuscolare del sole:

b) luce non aurorale. Essa è dovuta arli atomi o molecole eccitate per assorbimento di fotoni. Si suppone che durante il giorno la radiazione solare dissoci le molecole in atomi (o li ionizzi) e che di notte esse si riformino (o tornino neutri), ovviamente allo stato eccitato, e quindi si abbia l'emissione di radiazioni;

c) luce del crepuscolo. Al crepuscolo si osserva che alcune righe spettrali mantengono una intensità abbastanza elevata; cito per es. il doppietto giallo del sodio e la linea rossa dell'idrogeno. Essi sono dovuti o ad una diffusione della radiazione per risonanza (easo del sodio) o ad una vita media molto lunga dello stato eccitato (caso dell'azoto atomico per la Iunghezza d'onda $\lambda=5200 \AA$ ).

Senza entrare nei dettagli dei problemi accennati, ciò che mi allontanerebbe molto dalla meta prefissatami, accemerò solamente al alcune questioni ad esse relative.

Le ricerche sono principalmente indirizzate verso i seguenti argomenti:

I) individuazione delle righe emesse. In questo modo si possono determinare i componenti dell'atmosfera, il loro stato di aggregazione e 
di ionizzazione. Questo problema viene affrontato sperimentalmente con degli spettrografi e luminosità molto elevata. Le aperture sono dellordine di $1: 0,4$ con lunghezze dello spettro visibile intorno a qualche millimetro e con dispersioni dell'ordine di qualche centinaio di angstrom per mm. Questa elevata luminosità è richiesta dal fatto che la brillanza del cielo notturno è molto bassa: si ha infatti sulla terra un illuminamento dell'ordine di $10^{-4} \mathrm{lux}$, contro i $10^{-1}$ lux dovuti alla luma piena ed i $10^{5} \mathrm{lux}$ dovuti al sole, allo zenith.

II) Righe proibite. Nello spettro della luce del dielo notturno si sono osservate delle righe corrispondenti a transizioni cosidette proibite. Sono delle transizioni non osservabili nelle condizioni ordinarie di laboratorio, quando ciò̀ si opera con pressioni relativamente elevate. Ciò è dovuto al seguente fenomeno. Un elettrone di un atomo eccitato può trovarsi in uno dei tanti livelli energetici: ogni livello ha ma vita media, nel senso che l'elettrone può rimanervi per un certo periodo di tempo che può avere un valore molto diverso da livello a livello. Cosi per le righe osservate in laboratorio può essere dell'ordine di $10^{-8}$ sec., mentre in altri casi può essere molto più lungo e cioè dell'ordine del secondo e per"fino dell'or'a.

Nel primo caso, se la pressione del gas è sufficientemente elevata e cioè se il eammino libero medio dell'atomo è abbastanza piceolo, l'elettrone tormera allo stato fondamentale prima che l'atomo abbia subito degli urti con gli atomi (o molecole) vicini. Nel secondo caso l'atomo subirà un numero più o meno grande di urti che faranno passare l'energia dovuta allo stato ecceitato agli altri atomi (o molecole) sotto forma di emergia cinetica. Ma se il ammino libero merlio è abbastanza grande, in modo che il tempo intercorso tra due urti successivi sia dello stesso ordine di grandezza della vita media del livello, allora l'atomo potrà emettere la radiazione prima di ceedere la sua energia agli altri atomi.

Questi livelli a vita molto lunga si chiamano stati metastabili: nella Fig. 5 sono riportati quattro casi cli livelli con le relative vite medie.

A3. - Determinazione della quota degli strati di emissione. Anche qui ci troviamo di fronte alle difficoltà dello stesso tipo di quelle accennate nel caso degli spettri di assorbimento. Il metodo più usato è quello di Van Rijn che presuppone uno strato sottile e uniforme e che si basa su due misure di intensità a due diverse distanze zenitali. Con l'ipotesi rhe l'intensità della radiazione emessa sia semplicemente proporzionale allo spessore che lo struto presenta nella direzione di osservazione, il 
problema diventa puramente geometrico, analogo a quello già trattato nel caso dell'assorbimento (v. Fig. 2). Anche qui si ha

$$
I=\sqrt{\frac{I_{0}}{1-\left(\frac{R}{R+H}\right)^{2} \operatorname{sen}^{2} \zeta}}
$$

dove $I_{0}$ ed $I$ sono le intensità osservate allo zenith e in una direzione generica a distanza zenitale $\zeta$.
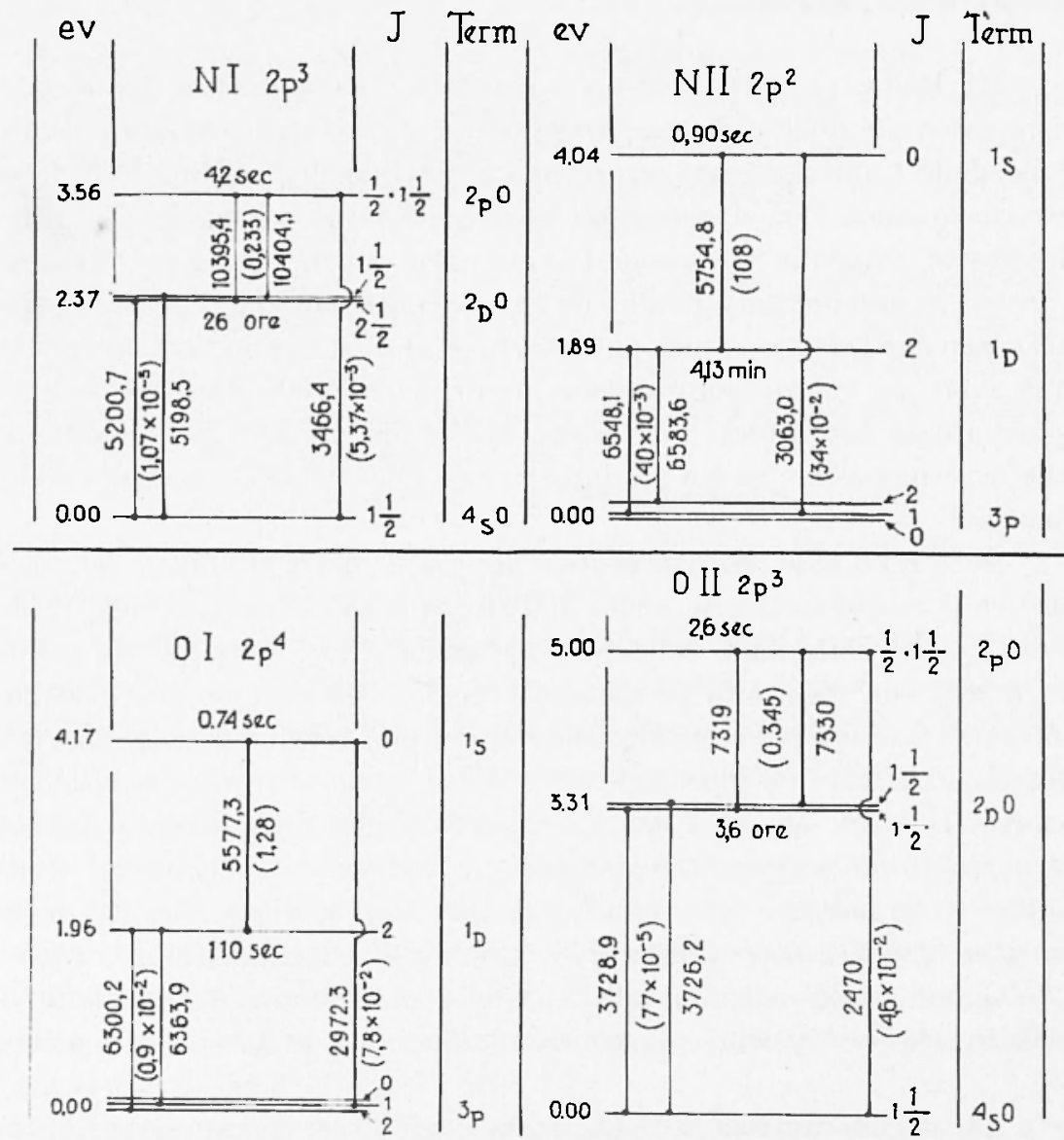

Fig. $5(*)$

Un altro metodo sviluppato in questi ultimi anni è quello basato sulla misura, eseguita per mezzo di razzi, della intensità delle righe emesse.

(*) Da J. W. Chamberlain e A. B. Meinel in " The Earth as a planet $\left({ }^{3}\right)$ p. 527 . 
Riporto nella Fig. 6 il principio nel quale si basa questo metodo e nella Fig. 7 i risultati ottenuti relativi a tre componenti dell'atmosfera.

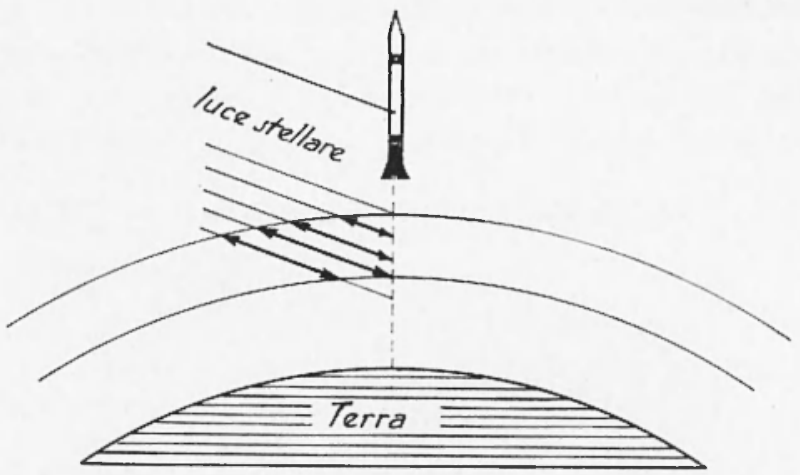

Fig. $6(*)$
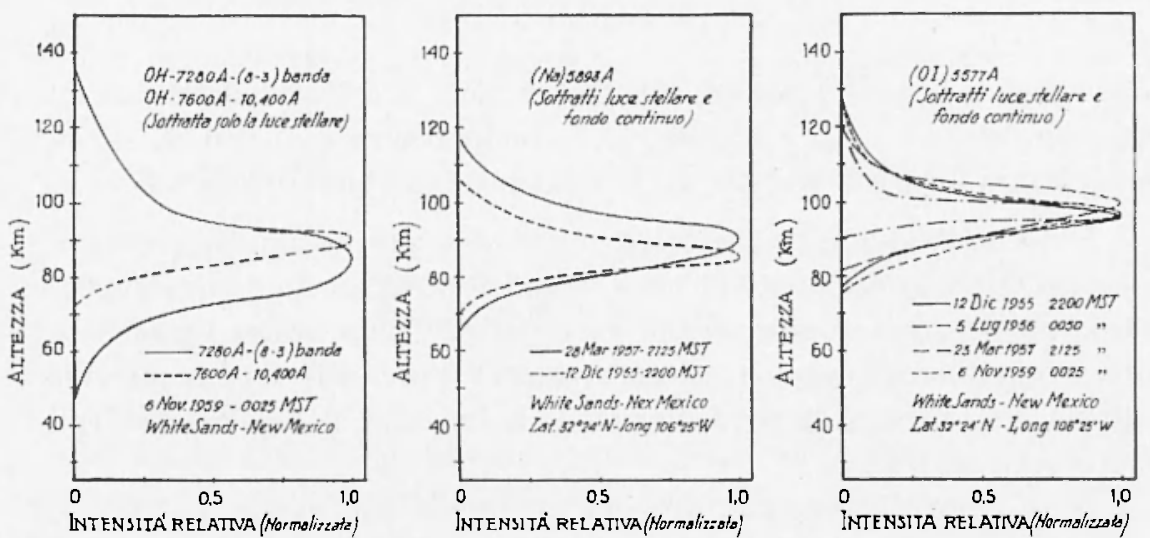

Fig. $7(* *)$

A4. - Determinazione della temperatura dell'atmosfera alla quota dello strato emittente. Due sono i metodi adoperati. Il primo consiste nella misura dell'allargamento per effetto Doppler di alcune righe particolarmente sottili: si usa per es. la riga dovuta alla transizione proibita ${ }_{2}^{3}[O I]$ avente la lunghezza d'onda $\lambda=5577 \AA$. Questo metodo ha prima di tutto il difetto di presupporre una distribuzione maxwelliana delle velocità degli atomi eccitati, ciò che non sempre è vero in quanto essi provengono da una fotodissociazione e la loro densità è relativamente

(*) Da H. Friedman in "Space Research" $\left(^{5}\right)$ p. 1031.

$(* *)$ Da H. Frieduan in "Space Research" $\left(^{5}\right)$ pp. 1032, 1033. 
bassa. Il metodo presenta anche il difetto di non poter separare le velocità dovute all'agitazione termica da quelle dei moti turbolenti dell'atmosfera, velocità che sono dello stesso ordine di grandezza.

Un metodo molto più preciso è quello basato sul fatto che l'intensità delle righe di una banda molecolare risentono molto della temperatura. Cosi per es. per la banda $N_{\underline{2}}^{+}$di lunghezza d'onda $\lambda=391.1 \times$ (Fig. 8)

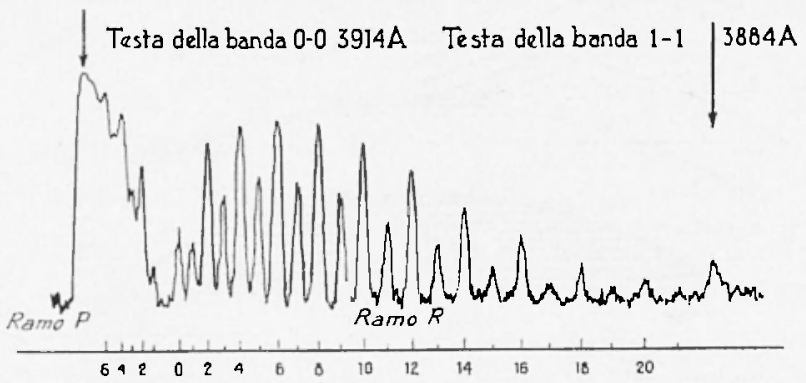

Fig. $8\left({ }^{*}\right)$

si vede che i successivi massimi relativi al ramo $R$ della banda hamno un massimo intorno a $K=6$, quando la temperatura d di 250 oK; se la temperatura fosse salita a 490 oK, il massimo si sarebbe trovato a $K=8$.

B. - Lo studio dello spettro u.v. e X del sole ha una importanza notevole non solo dal punto di vista astrofisico, ma anche da quello geofisico. Infatti sono queste regioni spettrali che influenzano fortemente tutti gli equilibri tara atomi e molecole (neutri ed eccitati) dei gas presenti nell'alta atmosfera ed in particolar modo influenzano la formazione degli strati della ionosfera.

Dato l'elevato assorbimento dell'atmosfera per queste radiazioni, nessun mezzo ci permette l'osservazione eccettuato i razzi ed i satelliti. I metodi adoperati sono: gli spettrografi, con i quali si è arrivati fino a $500 \AA$, a quote intorno ai $200 \mathrm{~km}$ e monoclomatori con cellule fotoelettriche o contatori di fotoni, con i quali si è arrivati fino a $60 \AA$, alla quota di $198 \mathrm{~km}$.

Desidero aceennare rapidamente al fatto che sono state osservate delle sorgenti intense di radiazione u.v. in alcune stelle ed in alcune nebulosità in vicinanza di stelle calde.

C. - Il terzo campo di ricerehe da me esaminato riguarda la diffusione della luce da parte dell'atmosfera. Questo fenomeno è molto dannoso in p. 538 .

(*) Da J. W. Chamberdain e M. Meiner in "The Earth as a planet " $\left.{ }^{3}\right)$ 
particolar modo per la visione di oggetti extraatmosferici molto deboli, quali le stelle e la corona solare. Neanche a quote relativamente elevate essa può essere eliminata, in quanto la brillanza del cielo è approssimativamente proporzionale alla massa dell'aria e quindi alla pressione: cosi per es. a $11.600 \mathrm{~m}$ la brillanza è ridotta a un quinto della brillanza al livello del suolo. Perché la brillanza sia ridotta a 1/100 occorre salire ad una quota di $31 \mathrm{~km}$.

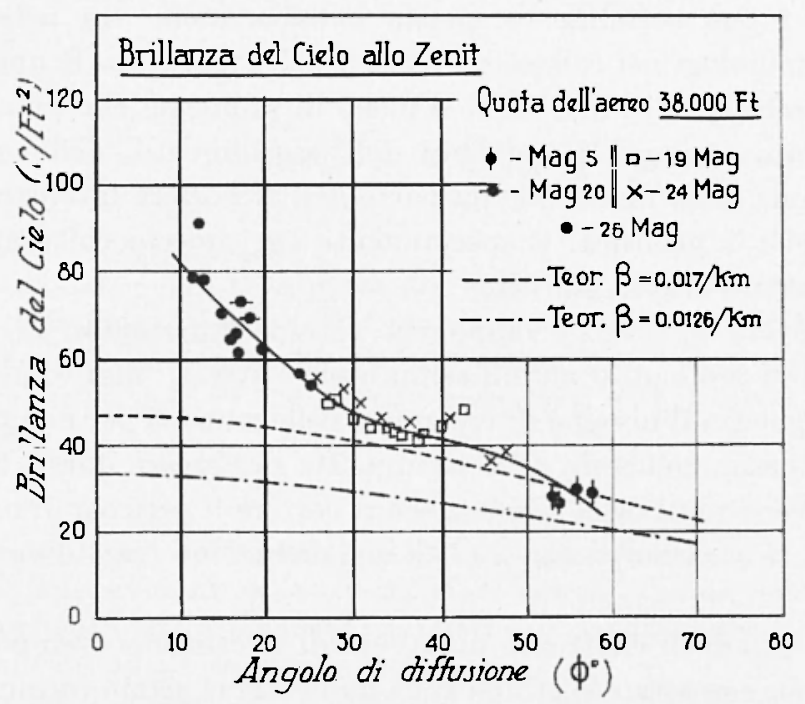

Fig. $9\left({ }^{*}\right)$

Lo studio della luce diffusa è perciò molto importante sia per gli astronomi sia per i geofisici. Infatti la luce diffusa risente molto della purezza dell'atmosfera, cioè dipende dal numero e dalla qualità di centri diffusori presenti. Infatti la diffusione alla Rayleigh presuppone che questi diffusori siano solo molecole; in realtà nell'atmosfera essi sono formati da aggregati molecolari o da particelle liquide o solide. Nella Fig. 9 sono riportate alcune misure eseguite in giornate particolarmente serene nell'Arizona ad una quota di $11.600 \mathrm{~m}$ : l'aumento notevole di intensità in vicinanza di $\varphi=0$, denuncia l'esistenza di diffusori grossi.

In questo quadro di ricerche si è inserito un gruppo di lavori promossi dall'Osservatorio Astronomico su M. Mario, dall'Istituto Nazionale di Geofisica e dal Ministero della Difesa Aeronautica, tendente a studiare la possibilità di fotografare gli oggetti celesti da quote intorno ai $20-30 \mathrm{~km}$.

(*) Da 1. M. PACKER e (. LOCK $\left(^{8}\right)$ p. 476. 
Questo gruppo di lavori è stato iniziato con il lancio di un pallone che portava una macchina fotografica del tipo all-sky; il lancio avvenne a Pisa durante l'eclisse totale di sole del 15-2-1961. L'illuminazione eccezionale dell'atmosfera terrestre durante un'eclisse totale ha permesso di fare delle notevoli osservazioni sulla diffusione della luce da parte dell'alta atmosfera. Ora si sta continuando questa ricerea allo scopo di trovare le condizioni migliori per la fotografia diuma di oggetti celesti molto deboli.

Desidero infine sottolineare questa collaborazione tra astronomi, geofisici e meteorologi nel campo dell'ottica dell'atmosfera. E questo un campo di ricerche quanto mai vasto e pieno di promesse che puó e deve essere aflrontato insieme: i problemi dell'assorbimento, dell'emissione e della diffusione della radiazione da parte dell'atmosfera interessano sia l'astronomia che la geofisica. E specialmente oggi questa collaborazione assume un particolare significato.

Mai come ora Ie scienze vamno via via specializzandosi in infiniti rami sempre più profondi e quindi sempre più ristretti: mai come ora si sente di conseguenza il bisogno di creare dei collegamenti per non pertere di vista il panorama genelale della natura. Ma per creare questi collegamenti tra i diversi rami della scienza, senza correre il pericolo di minarne la consistenza, è necessaria una stretta collaborazione tra i diversi specialisti.

Si tratta quindi di effettuare un lavoro di ricerea e contemporaneamente di sintesi; e questa è la st rada sulla quale noi ci siamo incamminati con queste prime ricerche. La meta finale, come in tutte le ricerche, è molto lontana e probabilmente non sarà mai raggiunta; ma essa per noi rappresenta la speranza e la gioia delle nostre fatiche.

\section{BIBLIOGRAFIA}

Tra i numerosissimi lavori sullargomento citerò solo alcuni, che mi sono apparsi particolarmente significativi sia per la visione d'insieme che per la particolarití della ricerca.

(1) R. II. Goovr, The physics of the stratosphere. Cambridge, 1954.

(2) II.S. W. MAssey, R. I. F. BOYD, The upper atmosphere. Iondon, 1958.

(3) G. P. Kun'er, The Earth as a planet. Chicago 1954.

(") Hidide Kaldiann Bijl, Space Researeh, v. I, Amsteriam 1960.

(5) II. C. vax de IIUls', C. DE JAGER, A. F. MoOre, Space Researeh. v. II, Amsteriam, 1961.

${ }^{\left({ }^{6}\right)}$ J. C. Johnson, Phisical Meteorolog!y. Cambrilge, 1954.

(7) H. F. Newer., IIigh Altitude Rocket Research. New York, 1953.

$\left.{ }^{8}\right)$ J. A. Ratclifie, Physies of the upper atmosphere. New York, 1960.

( ${ }^{\circ}$ D. IL. PACker, C. Lock, J. of Optical Soc. of America, 41, 473 (1951).

(10) R. Gialdea, M. Cimino, G. Fes, Ann. di Gieofisica, XV, 79 (1962). 